\title{
THE BEHAVIOR OF INVERTED PENDULUM STRUCTURES DURING EARTHQUAKES
}

By George W. Housner

\begin{abstract}
During the Chilean earthquakes of May, 1960, a number of tall, slender structures survived the ground shaking whereas more stable appearing structures were severely damaged. An analysis is made of the rocking motion of structures of inverted pendulum type. It is shown that there is a scale effect which makes tall slender structures more stable against overturning than might have been expected, and, therefore, the survival of such structures during earthquakes is not surprising.
\end{abstract}

\section{INTRODUCTION}

During the Chilean earthquakes of May, 1960, several golf-ball-on-a-tee types of elevated water tanks survived the ground shaking despite the appearance of instability, as described in a companion paper by W. K. Cloud. On the other hand, much more stable-appearing reinforced-concrete, elevated water tanks were severely damaged as described in a companion paper by $K$. V. Steinbrugge and $R$. Flores. It is clear that, in this case, the concept of representing the effect of an earthquake by a certain static lateral force (percent $g$ design) may be quite misleading. It is not uncommon for building codes to specify that elevated water tanks should be designed for 10 per cent $g$ and, judged from this point of view, the golf-ball tank appears to be in danger from earthquakes whereas the strength and proportions of the concrete tank would be reassuring as to its ability to survive earthquakes. Other unstable types of structures have also survived strong earthquakes. During the Arvin-Tehachapi earthquake in California (July 21, 1952) a number of tall, slender, petroleum-cracking towers stretched their anchor bolts and rocked back and forth on their foundations. ${ }^{1}$ In some Indian cities, free-standing stone columns that supported heavy statues remained standing although at the end of the earthquake they were surrounded by heaps of debris that had been more stable structures. Tall, slender stone pillars in graveyards have also survived strong ground motions. On the other hand, it has sometimes happened that box-like electric power transformers have rocked and overturned. Some light is thrown on this anomalous behavior by an analysis of the dynamics of a rigid block that is resting upon a rigid, horizontal base and is excited into rocking motion. It will be seen that the vibrational characteristics of this type of structure are markedly different from those of a linearly elastic structure.

\section{Free Vibrations}

The rigid block shown in fig. 1 will oscillate about the centers of rotation 0 and $0^{\prime}$ when it is set to rocking. It is assumed that the coefficient of friction is sufficiently large so that there will be no sliding between the block and the base. The significant properties of the block are its weight, $W$, its moment of inertia, $I_{0}$, about

\footnotetext{
${ }^{1}$ Housner, G. W., "Limit Design of Structures to Resist Earthquakes," Proceedings of the 1956 World Conference on Earthquake Engineering, Earthquake Engineering Research Institute, 1956. 
the point 0 , and the location of the center of gravity a distance $h$ above the base and a distance $b$ from the side of the block. As shown in fig. 1, the radial distance from the center of rotation 0 to the center of gravity is $R=\sqrt{h^{2}+b^{2}}$. When the block is at rest, the line $R$ makes an angle $\alpha$ with the vertical. The tilting of the block from the vertical is measured by the angle $\theta$.

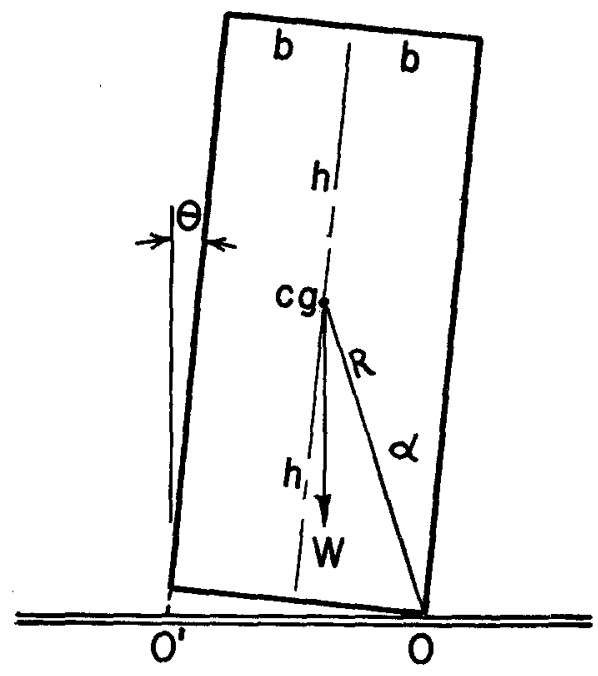

FIG. 1. A rocking block.

When the block is rotated through an angle $\theta$, the weight of the block will exert a restoring moment $W R \sin (\alpha-\theta)$. The equation of motion is then

$$
I_{0} \frac{d^{2} \theta}{d t^{2}}=-W R \sin (\alpha-\theta)
$$

For tall, slender blocks having the angle $\alpha$ less than about $20^{\circ}$ the sine of the angle may be approximated by the angle and equation (1) may be written

$$
I_{0} \ddot{\theta}-W R \theta=-W R \alpha
$$

This equation describes the free vibration of the block. Setting $W R / I_{0}=p^{2}$, the equation becomes

$$
\ddot{\theta}-p^{2} \theta=-p^{2} \alpha
$$

This equation, subject to the conditions $\theta=\theta_{0}$ and $\dot{\theta}=0$ at $t=0$ which represent the block released from rest with initial displacement $\theta_{0}$, has the solution:

$$
\theta=\alpha-\left(\alpha-\theta_{0}\right) \cosh p t
$$

Equation (2) describes the rotation of the block about the point 0 as it falls back into the vertical position. The block will then tilt about the point $0^{\prime}$, and if there is negligible energy loss during impact, the block will rotate through an angle $\theta=$ $-\theta_{0}$. The block will then fall back again to the vertical position and will rise about point 0 until $\theta$ is again equal to $\theta_{0}$. At this instant, one complete cycle will have been completed. The time, $T$, required to complete this cycle is the period of free vibra- 
tion. The block will fall from $\theta=\theta_{0}$ to $\theta=0$ in a time $t=T / 4$ and at this instant equation (2) becomes

$$
0=\alpha-\left(\alpha-\theta_{0}\right) \cosh p \frac{T}{4}
$$

or

$$
\cosh \frac{p T}{4}=\frac{1}{1-\theta_{0} / \alpha}
$$

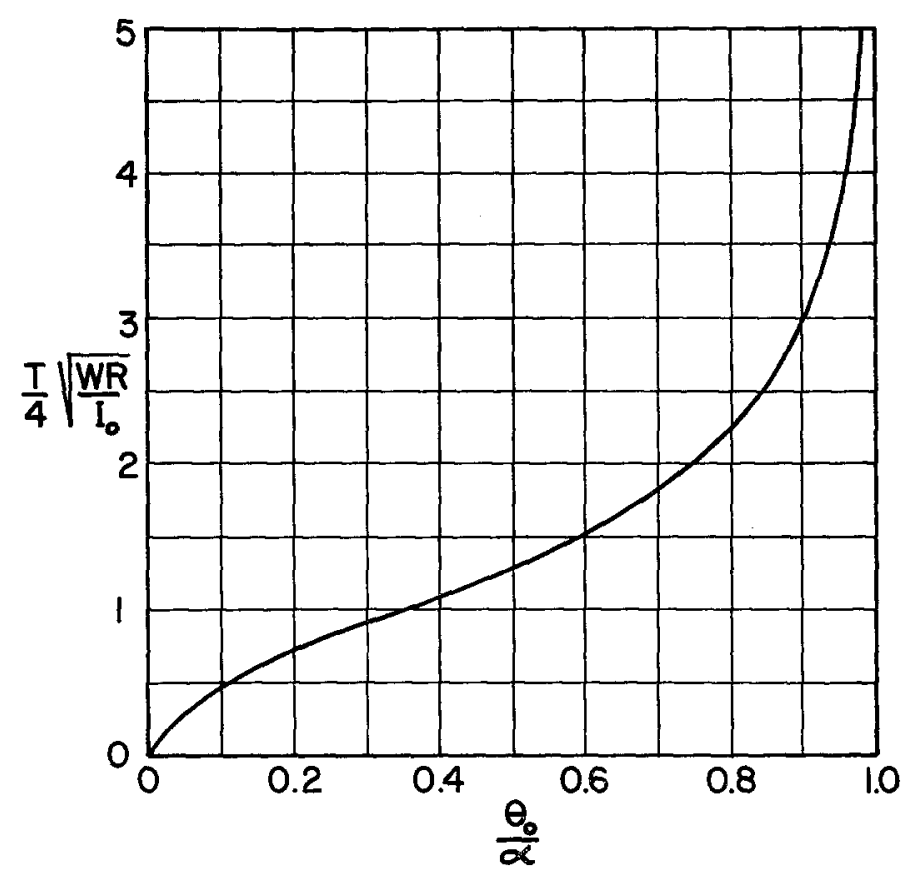

FIG. 2. Period $T$ of block rocking with amplitude $\theta_{0}$.

Equation (3) expresses the period $T$ in terms of $p$ and $\theta_{0} / \alpha$

$$
T=\frac{4}{p} \cosh ^{-1}\left(\frac{1}{1-\theta_{\mathrm{c}} / \alpha}\right)
$$

A graph of this equation is shown in fig. 2 where it is seen that the period is strongly dependent upon the amplitude ratio $\theta_{0} / \alpha$. When $\theta_{0} / \alpha$ is close to unity the period is long, and when $\theta_{0} / \alpha$ is close to zero the period is short.

During the rocking of a real block there would be, of course, a dissipation of energy when the block impacted on the base. Thus, once during each half-cycle there would be an increment decrease in the energy of vibration. Consequently, the period of each half-cycle would be longer than that which preceded it. If the impact is assumed to be inelastic (no bouncing), the rotation continues smoothly about the point $0^{\prime}$ and the moment of momentum about $0^{\prime}$ is conserved. The reduction in kinetic energy during impact is

$$
r=\left(\frac{1}{2} I_{0} \dot{\theta}_{2}^{2}\right) /\left(\frac{1}{2} I_{0} \dot{\theta}_{1}^{2}\right)=\left(\frac{\dot{\theta}_{2}}{\dot{\theta}_{1}}\right)^{2}
$$


Equating the moment of momentum about $0^{\prime}$ immediately before impact to that immediately after impact gives

$$
I_{0} \dot{\theta}_{1}-2 m R b \dot{\theta}_{1} \sin \alpha=I_{0} \dot{\theta}_{2}
$$

This gives for the reduction of energy by the impact

$$
r=\left[1-\frac{m R^{2}}{I_{0}}(1-\cos 2 \alpha)\right]^{2}
$$

For slender blocks this relation may be written

$$
\sqrt{r}=1-\frac{2 m R^{2} \alpha^{2}}{I_{0}}
$$

Using the notation $\varphi=\theta / \alpha ; \varphi_{0}=\theta_{0} / \alpha$, the displacement and velocity of the block may be written

$$
\begin{aligned}
& \varphi=1-\left(1-\varphi_{0}\right) \cosh p t \\
& \dot{\varphi}=-\left(1-\varphi_{0}\right) p \sinh p t
\end{aligned}
$$

At the instant of the $n$-th impact $\left(\varphi=0, t=t_{n}\right)$

and

$$
\cosh p t_{n}=\frac{1}{1-\varphi_{n-1}}
$$

$$
\sinh ^{2} p t_{n}=\left(\frac{1}{1-\frac{\varphi_{n-1}}{1-}}\right)^{2}-1
$$

Using this relation, the impact velocity may be written:

$$
\dot{\varphi}_{n}=-p \sqrt{1-\left(1-\varphi_{n-1}\right)^{2}}
$$

where $\varphi_{n}$ is the amplitude following the $n$-th impact. The kinetic energy just after impact is reduced to $r$ times the energy just before impact. The velocity is therefore reduced to $\sqrt{r}$ times the velocity before impact. This condition may be written

$$
-p \sqrt{1-\left(1-\varphi_{n}\right)^{2}}=-p \sqrt{r} \sqrt{1-\left(1-\varphi_{n-1}\right)^{2}}
$$

This equation can be written

$$
1-\left(1-\varphi_{n}\right)^{2}=r\left[1-\left(1-\varphi_{n-1}\right)^{2}\right]
$$

and, as this recurrence relation implies,

$$
1-\left(1-\varphi_{n}\right)^{2}=r^{n}\left[1-\left(1-\varphi_{0}\right)^{2}\right]
$$

Solving this for $\varphi_{n}$ gives

$$
\varphi_{n}=1-\sqrt{1-r^{n}\left[1-\overline{\left.\left(1-\varphi_{0}\right)^{2}\right]}\right.}
$$

A graph of this equation for $r=0.7$ is presented in fig. 3 where the decrease in amplitude for successive $n$ is shown for several values of the initial displacement. 
It is seen that for large amplitudes the energy of vibration decreases rapidly but for small oscillations the energy decreases slowly.

It follows from equations (3) and (6) that the successive half periods of vibration during the free rocking are given by

$$
\frac{T}{2}=2 \sqrt{\frac{I_{0}}{W R}} \tanh ^{-1} \sqrt{r^{n}\left[1-\left(1-\varphi_{0}\right)^{2}\right]}
$$

According to the foregoing analysis the free rocking of a block proceeds as follows. At first there will be a few oscillations of large amplitude and slow rocking. The amplitude decreases markedly with each impact and corresponding with this there is an increase in frequency of oscillation to an infinite value. Actually, a freely rocking block does not make a truly inelastic impact, but there is a slight bouncing and sliding involved. This is another source of energy absorption and under favor-

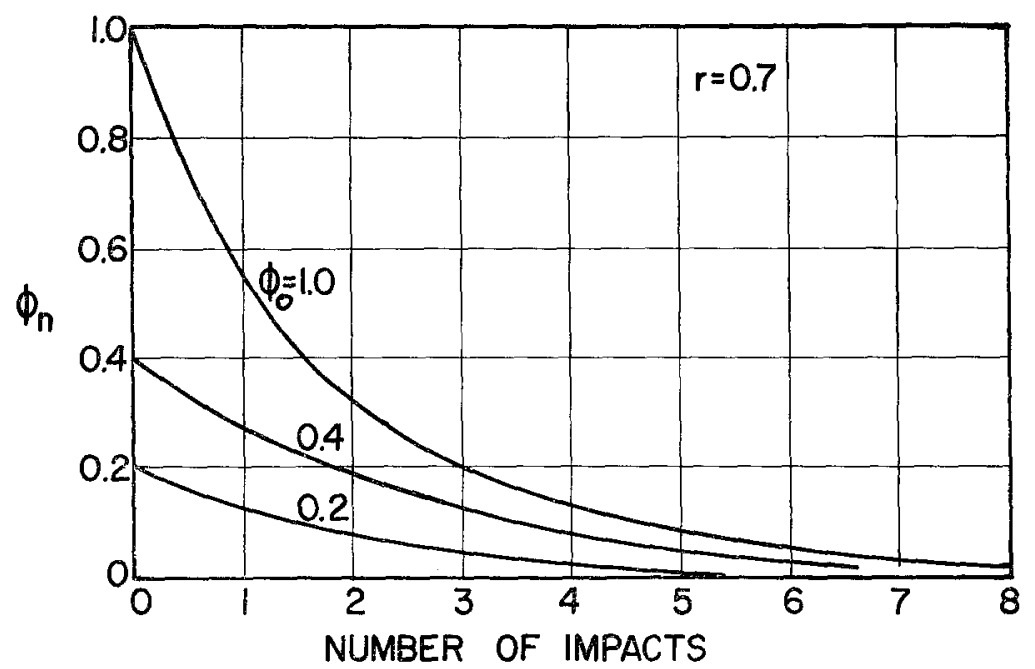

FIG. 3. Amplitude $\varphi_{n}$ subsequent to $n$-th impact.

able conditions the block makes only some eight or ten impacts before being brought to rest.

\section{Overturning by Constant Acceleration}

If the block is resting on a base which is suddenly given a constant acceleration $a$ lasting for a time $t_{1}$ the block may or may not overturn, depending upon the magnitude of $a$ and the duration of $t_{1}$. For small angles of oscillation the effect of the acceleration is the same as if a force $W a / g$ were acting horizontally through the center of gravity of the block and thus the equation of motion is:

$$
I_{0} \ddot{\theta}-W R \theta=W R a / g-W R \alpha
$$

It is seen from this equation that a necessary condition for motion to be initiated is that $a / g>\alpha$, which specifies the fraction of $g$ acceleration required to begin tilting 
the block. The solution of equation (7), subject to the conditions that at $t=0$ both the velocity and displacement are zero is

$$
\begin{aligned}
\frac{\theta}{\alpha} & =\left(\frac{a \alpha}{g}-1\right)(\cosh p t-1) \\
p & =\sqrt{\frac{\overline{W R}}{I_{0}}}
\end{aligned}
$$

It is seen that if $a / g>\alpha$ and if the acceleration acts for a sufficient length of time, the block will overturn. It is not necessary, however, for the base acceleration to act continuously in order to overturn the block. For each value of $a$ there is a time $t_{1}$ during which the acceleration can generate a velocity sufficient to overturn the block.

The condition for overturning is that the total work done by the inertial force $W a / g$ is just equal to the difference in potential energy between positions $\theta=\alpha$ and $\theta=0$. This condition may be written

$$
\int_{0}^{t_{1}} R W(a / g) \dot{\theta} d t=W R(1-\cos \alpha)
$$

Carrying out the integration and approximating $(1-\cos \alpha)$ by $\alpha^{2} / 2$, equation (8) becomes

$$
\frac{a}{g}\left(\frac{a}{g} \alpha^{2}-\alpha\right)\left(\cosh p t_{1}-1\right)=\frac{\alpha^{2}}{2}
$$

If this equation is solved for $\cosh p t_{1}$, there is obtained

$$
\cosh \sqrt{\frac{W R}{I_{0}}} t_{1}=1+\frac{1}{2 \frac{a}{g \alpha}\left(\frac{a}{g \alpha}-1\right)}
$$

Figure 4 is a graph of this equation giving the duration, $t_{1}$, of ground acceleration $a$ required to overturn the block.

The foregoing analysis is not realistic for earthquake ground motions since it assumes constant ground accelerations of finite duration followed by a constant velocity of the ground. This type of ground motion does not occur during earthquakes and hence it is not meaningful to discuss the overturning of blocks in terms of percent $g$ acceleration.

\section{Overturning by Sinusoidal Acceleration}

The accelerograms recorded during earthquakes show certain maximum peaks whose shapes can be approximated closely by one loop of a sine wave. The question then arises as to the amplitude and period of a half sine-wave acceleration pulse that will suffice to overturn the block.

If the base is given a sinusoidal acceleration, the equation of motion is

$$
I_{0} \ddot{\theta}=-W R \sin (\alpha-\theta)+W R \frac{a}{g} \sin (\omega t+\psi)
$$


In this equation the ground acceleration has been expressed by

$$
-a \sin (\omega t+\psi)
$$

where $a$ is the amplitude of the acceleration and $\psi$ is defined by the condition

$$
a \sin \psi=g \sin \alpha
$$

This insures that at time $t=0$ the base acceleration will have reached the value required to initiate rocking of the block. For small angles, and setting $W R / I_{0}=$ $p^{2}$, the equation may be written:

$$
\ddot{\theta}-p^{2} \theta=\alpha p^{2}\left[\frac{\sin (\omega t+\psi)}{\sin \psi}-1\right]
$$

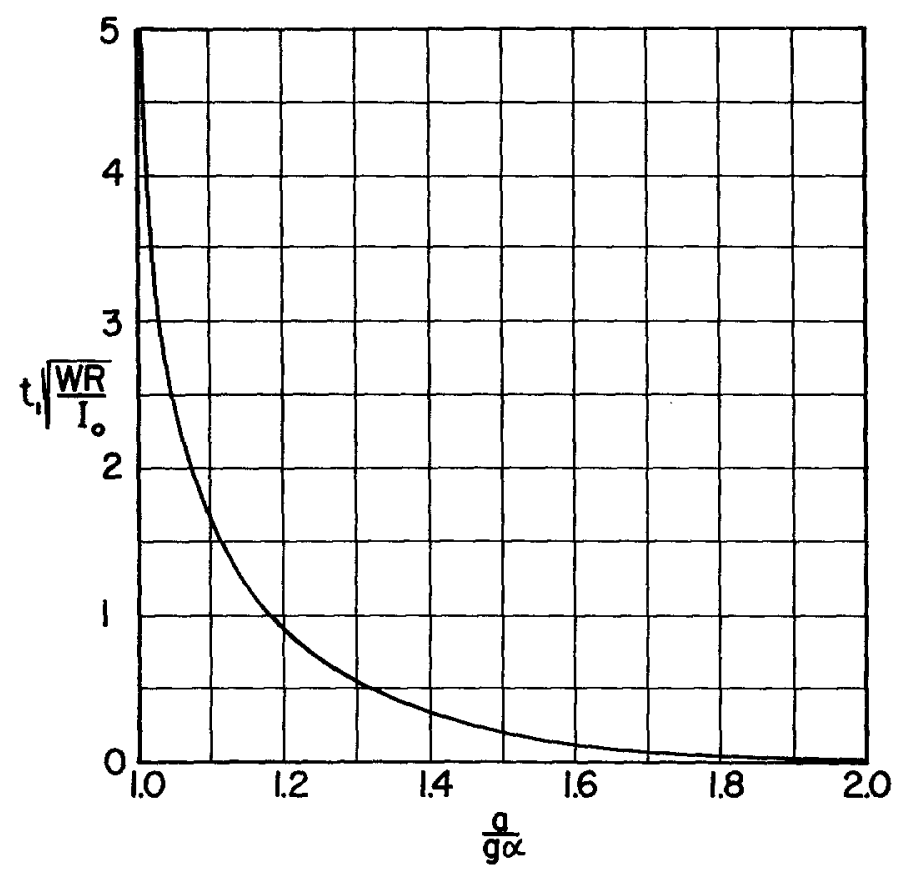

FIG. 4. Constant acceleration $a$ of duration $t_{1}$ required for overturning.

The solution of this equation, subject to the conditions $\theta=0, \dot{\theta}=0$ at $t=0$, is

$$
\frac{\theta-\alpha}{\alpha}=\frac{1}{1+\left(\frac{\omega}{p}\right)^{2}}\left[\frac{\omega}{p} \frac{\sinh p t}{\tan \psi}-\left(\frac{\omega}{p}\right)^{2} \cosh p t-\frac{\sin (\omega t+\psi)}{\sin \psi}\right]
$$

where

$$
\sin \psi=g \alpha / a ; \quad \cos \psi=\sqrt{1-(g \alpha / a)^{2}}
$$

The condition for overturning is that $\theta=\alpha$ at time $t=(\pi-\psi) / \omega$. This insures that $\theta$ will reach the value $\alpha$ at the instant that the ground acceleration has completed its first half-cycle and has the value zero. 
Substituting in equation (10) and rearranging terms gives:

$$
\tan \psi=\frac{p}{\omega} \tanh \left[\frac{p}{\omega}(\pi-\psi)\right]
$$

and this equation specifies the amplitude and frequency of the sinusoidal acceleration pulse required to just overturn the block. Under the conditions of the problem tanh $p / \omega(\pi-\psi)$ may be approximated by unity and hence equation (11) may be written

$$
\tan \psi=\frac{p}{\omega}
$$

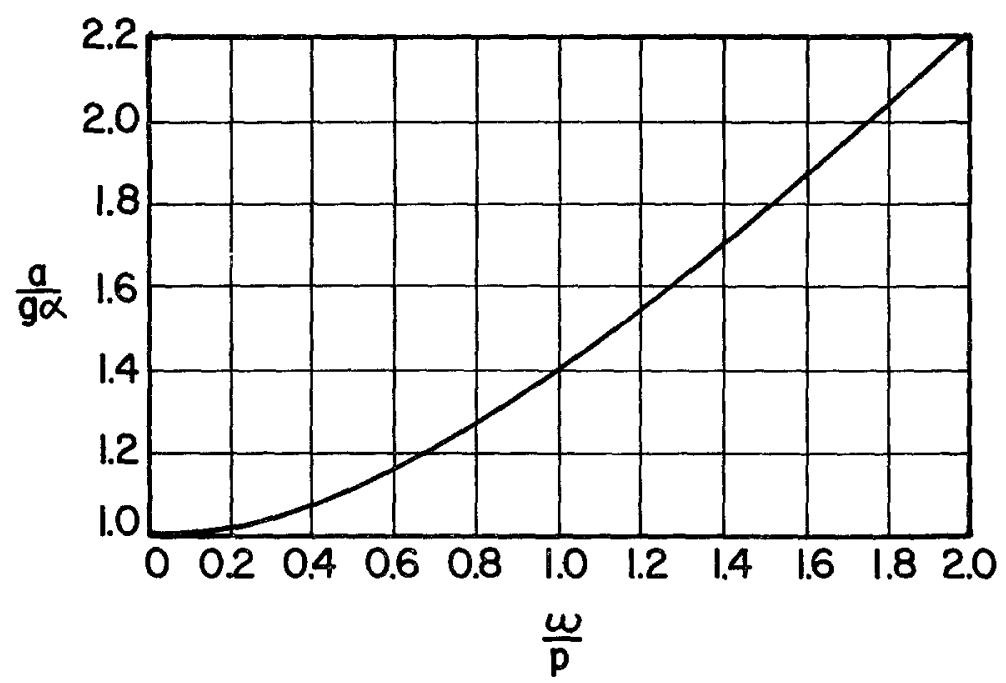

Frg. 5. Sinusoidal acceleration pulse $a \sin \omega t$ required for overturning.

Making use of equation (10) and rearranging terms, equation (11)a can be put in the form

$$
\frac{a}{g \alpha}=\sqrt{1+\left(\frac{\omega}{p}\right)^{2}}
$$

or

$$
\frac{a}{g \alpha}=\sqrt{1+\frac{I_{0}}{W R}\left(\frac{2 \pi}{T_{g}}\right)^{2}}
$$

where $T_{g}$ is the period of the ground acceleration. This relation was first derived by Kirkpatrick $^{2}$ and it expresses the conditions under which a single sine pulse will overturn the block. As may be verified, this is a minimum condition as it insures that $\dot{\theta}=0$ when $\theta=\alpha$.

A graph of equation (12) is presented in fig. 5 for small values of $\omega / p$. For large values of $\omega / p$, say $\omega / p>3$, equation (12) can be represented by

$$
\frac{a}{g \alpha}=\frac{\omega}{p}
$$

2 Kirkpatrick, P., "Seismic Measurements by the Overthrow of Columns," Bull. of the Seism. Soc. of Amer., Vol. 17, No. 2, 1927. 
or

$$
\frac{a}{g \alpha}=\frac{\pi}{\frac{T_{g}}{2} \sqrt{\frac{\overline{W R}}{I_{0}}}}
$$

As an example, a rectangular block such as shown in fig. 1 has

$$
\left(\frac{\omega}{p}\right)^{2}=\frac{4}{3} \frac{R \omega^{2}}{g}
$$

and hence equation (12)b can be written

$$
\frac{a}{g \alpha}=\sqrt{\frac{4}{3} \frac{\bar{R} \omega^{2}}{g}}
$$

or

$$
\frac{a}{\omega}=\alpha \sqrt{\frac{4}{3} R g}
$$

or

$$
\frac{a T_{g}}{2}=\alpha \cdot 2 \pi \sqrt{\frac{1}{3} R g}
$$

where $T_{\partial}$ is the period of the pulse. If we consider all blocks that have a given length of diagonal, $2 R$, the equation may be written

$$
\frac{a T_{g}}{2}=K \alpha
$$

where $K$ is a constant. It is thus seen that the product of the amplitude of the acceleration pulse by its duration must be proportional to the slenderness angle $\alpha$ for a given $R$.

\section{Overturning by Earthquake Motion}

The preceding paragraphs dealt with the overturning of the block by a square acceleration pulse and by a half sine-wave pulse and equations (9) and (12) give the minimum accelerations required to overturn a block by such single pulses. Smaller accelerations than are specified by these equations may set the block to rocking but will not overturn it. It is, however, possible to overturn the block with smaller accelerations if a number of pulses act successively. This would be the case of strong-motion earthquake ground acceleration, which may be thought of as a train of pulses which, in general, have periods that are short compared to the rocking periods of structures. The resulting behavior of the block is indicated by the following approximate analysis.

For purposes of illustration, consider ground acceleration composed of a sequence of $N$ discrete step changes $\pm \Delta v$ in ground velocity, that are randomly distributed over time $0<t<t_{1}$, and have equal probability of being positive or negative in sign. This represents an idealized earthquake ground motion whose average velocity response spectrum (undamped) is a constant. The effect of such ground motion on structures is the same as if the ground were at rest and impulsive inertial forces $F_{n} \Delta t$ were acting through the center of mass, where

$$
F_{n} \Delta t= \pm M \Delta v \quad(n=1,2 \cdots N)
$$


The response of a linearly elastic structure, such as is shown in fig. 6 , to the random succession of forces $F_{n}$ is to be excited into oscillations. The effect of the $n$-th impulse, $F_{n} \Delta t$, is to produce a change in velocity $\Delta v$, and hence to produce a change in kinetic energy of vibration

$$
\begin{aligned}
\Delta K E_{n} & =\frac{1}{2} M\left(v_{n}+\Delta v\right)^{2}-\frac{1}{2} M v_{n}^{2} \\
& =M v_{n}(\Delta v)+\frac{1}{2} M(\Delta v)^{2}
\end{aligned}
$$

where $v_{n}$ is the velocity of the structure at the time that $F_{n} \Delta t$ acts. If a large number of such idealized earthquakes were to act upon the structure, the $\Delta K E_{n}$ would,

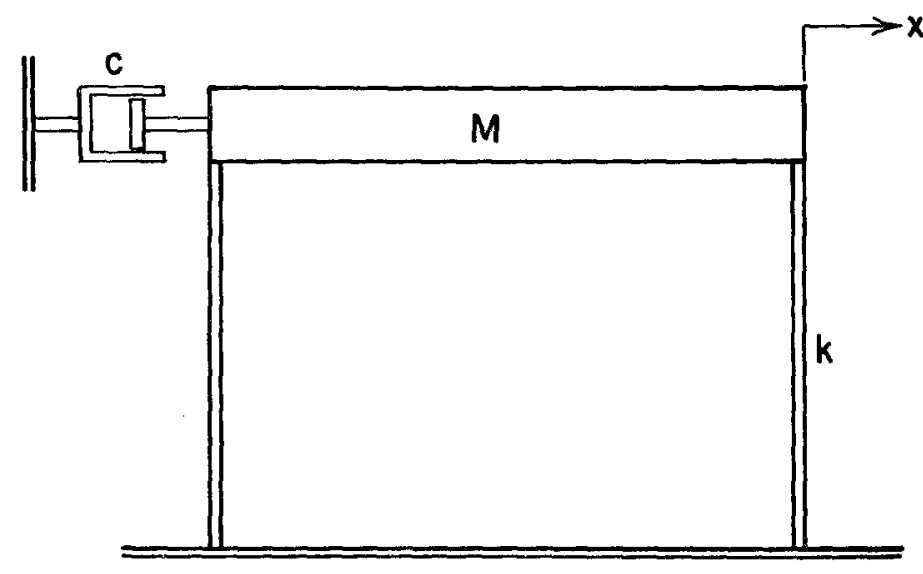

FIG. 6. Simple linear structure.

in general, be different for each of the earthquakes. However, the average increment change in energy, $\Delta K E_{n}$, averaged over all the earthquakes would be

$$
{\overline{\Delta K E_{n}}}_{n}=M{\overline{v_{n}(\Delta v)}}+\frac{1}{2} M(\Delta v)^{2}
$$

and since $\overrightarrow{v_{n}}$, the average velocity is zero and the average $\overline{\Delta v}$ is also zero, it follows that

$$
\overline{\Delta K \overline{E_{n}}}=\frac{1}{2} M(\Delta v)^{2}
$$

On the average, then, the total energy of vibration of undamped structures is

$$
\vec{E}_{n}=\frac{1}{2} M(\Delta v)^{2} n \quad(n=1,2 \cdots N)
$$

This represents an average building up of energy with the number of pulses and hence the average rate of energy input, $P$, is

$$
P=\frac{1}{2} M(\Delta v)^{2} \frac{N}{t_{1}}
$$

This constant power input $P$ will cause the average amplitude of vibration to increase proportional to $\sqrt{t}$.

If the structure has viscous damping, the power input, $P$, will build up the amplitude of vibration until the average rate of energy loss just balances the input. 
The energy loss per cycle for a linearly damped oscillator is $4 \pi \lambda E$, where $\lambda$ is the fraction of critical damping, and $E$ is the energy of vibration. The average time rate of energy loss is then $4 \pi \lambda \bar{E} / T$, where $T$ is the period of vibration. An energy balance for the system then is $^{3}$

$$
\frac{\partial \bar{E}}{\partial t}=P-\frac{4 \pi \lambda}{T} \bar{E}
$$

The solution of this equation is

$$
\bar{E}=\frac{P T}{4 \pi \lambda}\left(1-\exp \left(-\frac{4 \pi \lambda}{T} t\right)\right)
$$

The average energy of vibration thus increases asymptotically to $P T / 4 \pi \lambda$; however, for each individual earthquake the energy may be larger or smaller depending upon how the impulses happen to strike. For individual earthquakes there will be a certain probability distribution of $\mathrm{E}$ about the mean $\bar{E}$.

The behavior of a rocking block subjected to a series of random impulses is similar to that of the linearly elastic structure in that the impulses tend to build up the energy of vibration and hence the amplitude of the motion. However, as was shown in the analysis of the free vibrations, the energy dissipation of the block depends upon both the amplitude and frequency of rocking. In addition, if the amplitude of the block exceeds a limiting value the block will overturn. The effect of these differences are brought out by the following approximate analysis.

If the rocking block is subjected to the series of random pulses, the increment change in kinetic energy produced by $F_{n} \Delta t$ is

$$
\Delta K E_{n}=\frac{1}{2} I_{0}\left(\dot{\theta}_{n}+\Delta \dot{\theta}_{n}\right)^{2}-\frac{1}{2} I_{0} \dot{\theta}_{n}^{2}
$$

For slender bodies, having $I / I_{0} \simeq 1$, we may write approximately

$$
\begin{aligned}
I_{0} \Delta \dot{\theta}_{n} & =R F_{n} \Delta t \\
\Delta \dot{\theta}_{n} & =\frac{R F_{n} \Delta t}{I_{0}}=\frac{R M \Delta v}{I_{0}}
\end{aligned}
$$

and hence

$$
\Delta K E_{n}=M R(\Delta v) \dot{\theta}_{n}+\frac{1}{2} \frac{(M R)^{2}}{I_{0}}(\Delta v)^{2}
$$

and the ensemble average is

$$
\overline{\Delta K \overline{E_{n}}}=\frac{1}{2} \frac{(M R)^{2}}{I_{0}}(\Delta v)^{2}
$$

If there were no energy loss during the rocking, the buildup of energy would give on the average

$$
\bar{E}_{n}=\overline{\Delta K \overline{E_{n}}}=\frac{1}{2} \frac{(M R)^{2}}{I_{0}}(\Delta v)^{2} n
$$

\footnotetext{
${ }^{3}$ Hudson, D. E., "Response Spectrum Techniques in Engineering Seismology", Proceedings of the First'World' Conference on Earthquake Enginesring, Earthquake Engineering Research Institute, 1956.
} 
The total energy required to overturn the block is

$$
P E=W R(1-\cos \alpha)=\frac{W R \alpha^{2}}{2}
$$

On the average the condition for overturning at the end of $N$ pulses is

$$
\frac{W R \alpha^{2}}{2}=\frac{1}{2} \frac{(M R)^{2}}{I_{0}}(\Delta v)^{2} N
$$

or

$$
\alpha=\sqrt{\frac{\overline{M R^{2}}}{I_{0}} \frac{1}{R g}}(\Delta v) \sqrt{N}
$$

When the idealized earthquake motion satisfies equation (15) there is a 50 per cent probability that the ground motion will overturn the block. If $\alpha$ is greater than the quantity on the right side of equation (15) the probability of overturning is less than 50 per cent, and if $\alpha$ is smaller than this quantity the probability of overturning is greater.

If there is an energy loss during the rocking of the block, with a fraction $r E$ of the energy of vibration being lost at each impact on the base as described by equation (5), an energy balance would require

$$
\frac{\partial \bar{E}}{\partial t}=P-\frac{2 r \bar{E}}{T}
$$

with $T$, as defined by equation (4), given by

$$
T=\frac{4}{p} \cosh ^{-1}-\frac{1}{1-\frac{\theta_{0}}{\alpha}}
$$

The potential energy of the freely rocking block is

$$
P E=W R \alpha^{2}\left[\frac{\theta_{0}}{\alpha}-\frac{1}{2}\left(\frac{\theta_{0}}{\alpha}\right)^{2}\right]
$$

and for the forced vibration of the block the average instantaneous amplitude of rocking, $\theta_{0}$, is defined by

$$
\bar{E}=W R \alpha^{2}\left[\frac{\theta_{0}}{\alpha}-\frac{1}{2}\left(\frac{\theta_{0}}{\alpha}\right)^{2}\right]
$$

or

$$
\frac{\theta_{0}}{\alpha}=1-\sqrt{1-\frac{2 \alpha^{2} \bar{E}}{W R}}
$$

The energy balance can then be written

$$
\frac{\partial \bar{E}}{\partial t}=P-\frac{\operatorname{pr} \bar{E}}{2 \cosh ^{-1} \sqrt{1-\frac{2 \alpha^{2}}{W R} \bar{E}}}
$$


This may be compared with equation (13) for the elastic structure. For very small $\bar{E}$, the denominator of the right side approaches a value of 2 and equation (6) has essentially the same form as equation (13):

$$
\frac{\partial \bar{E}}{\partial t}=P-\frac{\operatorname{pr} \bar{E}}{2}
$$

On the other hand, when the amplitude of vibration approaches the unstable point $\left(\bar{E}=W R / 2 \alpha^{2}\right)$ the denominator becomes extremely large and the last term in equation (16) may be neglected and the equation written

$$
\frac{\partial \bar{E}}{\partial t}=P
$$

In this case the block is effectively undamped. It is thus seen that, although the equivalent fraction of critical damping remains constant, as the amplitude of rocking increases, the rate of energy dissipation decreases strongly and this in turn promotes an increased amplitude of vibration. It may, therefore, be expected that the behavior of the rocking block could be quite variable in that relatively small ground motion may fortuitously build up the amplitude at the beginning of the ground motion and lead to overturning the block.

\section{EfFect of Slenderness on Overturning}

The stability of a rocking type of structure may be estimated by comparing the energy input with the energy required to overturn the structure. If the energy input is computed from the velocity response spectrum, $S_{v}$, of the earthquake ground motion the requirement for overturning, equation (14), is

$$
\frac{1}{2} W R \alpha^{2}=\frac{1}{2} \frac{W}{g} \frac{M R^{2}}{I_{0}} S_{v}^{2}
$$

or

$$
\alpha=\frac{S_{v}}{\sqrt{g R}} \sqrt{\frac{M R^{2}}{I_{0}}}
$$

It is seen that this has the same form as equation (15). For slender structures $M R^{2} / I_{0}$ has a value close to unity so that equation (17) can be approximated by

$$
\alpha=\frac{S_{v}}{\sqrt{g R}}
$$

This equation may be interpreted as stating that for a given spectrum value $S_{v}$, a block having an angle $\alpha$ given by equation (17)a will have approximately a $50 \%$ probability of being overturned. The dimensions of the structure enter only through the length $R$ and as equation (17)a shows, the larger the value of $R$ the more stable against overturning is the block. That is, comparing two blocks of the same proportions, the larger block is more stable than the smaller. There is thus an unexpected scale effect which is explained by the fact that the ground motion is not scaled 
with the block. If equations (17)a, (9), and (12)a are compared, setting $M R^{2} / I_{0}=$ 1 and $\alpha=b / h$, there is obtained for small $\alpha$

$$
\begin{array}{lll}
\text { Earthquake motion: } & b=S_{v} \sqrt{\frac{h}{g}} \\
\text { Square pulse: } & b=\frac{a}{g}-\frac{2 h}{1+\sqrt{1+\frac{4 h}{g t_{1}^{2}}}} \\
\text { Sine pulse: } & b=\frac{a}{g} \frac{h}{\sqrt{1+\frac{h}{g}\left(\frac{2 \pi}{T_{g}}\right)^{2}}}
\end{array}
$$

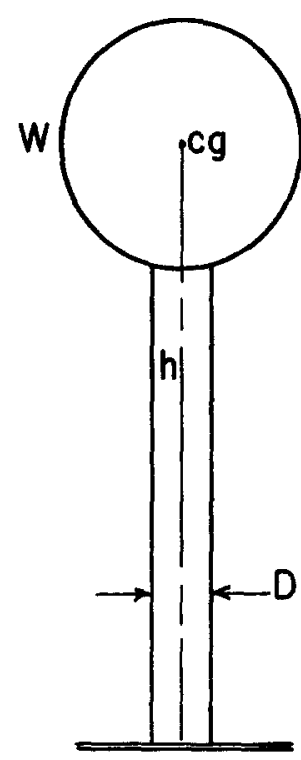

Fig. 7. Golf-ball-on-a-tee elevated water tank.

It is seen from these equations that the semiwidth $b$ required for stability does not increase linearly with $h$ as physical intuition indicates, but is affected less strongly, or in other words, tall slender structures are more stable than might be supposed.

\section{Golf-Ball Water Tank}

Let $h$ be the height of the center of gravity and $D$ the diameter at the base as shown in fig. 7, and suppose that the structure is rigid and is free to rock (no anchor bolts). For a structure of such proportions $h$ may be taken equal to $R$, and $I_{0}$ may be taken equal to $M R^{2}$. Equation (17) then becomes

$$
\alpha=\frac{D}{2 h}=\frac{S_{v}}{\sqrt{g h}}
$$

or

$$
S_{v}=\frac{1}{2} D \sqrt{\frac{g}{h}}
$$


This value of $S_{v}$ gives 50 per cent probability of overturning, and it is seen that the result is independent of the mass; that is, the stability is not affected by whether the tank is completely full or completely empty if $h$ may be taken to be a constant. Experience with earthquakes in the United States indicates that a value of $S_{v}=$ $2 \mathrm{ft} /$ sec represents strong ground motion. If the tank has $h=100 \mathrm{ft}$ and $D=10 \mathrm{ft}$, the required $S_{v}$ to give a 50 per cent probability of overturning is

$$
S_{v}=5 \sqrt{\frac{32.2}{100}}=2.6 \mathrm{ft} / \mathrm{sec}
$$

It may be concluded, therefore, that for an $S_{v}=2 \mathrm{ft} / \mathrm{sec}$, it is more likely that the tank would remain standing than that it would overturn. If the tank has anchor bolts that stretch and absorb energy during the rocking, there will be even less probability that the tank will overturn. It is, therefore, not so surprising that some golf-ball tanks have remained standing during earthquakes. There is, of course, a certain probability that a tank of these proportions will be overturned by a ground motion having $S_{v}=2.0$ or even less than 2.0 .

For comparison, suppose that the tank is tightly anchored to the foundation so that it cannot rock but can only bend and that the period of vibration is 3 seconds. In this case the maximum shear force generated would be

$$
F=\frac{2 \pi}{T} M S_{v}
$$

and

$$
\frac{F}{W}=\frac{2 \pi}{T} \frac{S_{v}}{g}=\frac{2 \pi}{3} \frac{2}{32.2}=0.13
$$

Thus the tank should be designed for 13 per cent $g$. It might appear from this that it would be advantageous to design tall slender structures to act as rocking blocks; however, a 50 per cent probability of survival for a rocking structure is not a satisfactory design. At present it is not known how to design a rocking structure to have specified small probability of failure. It may also be noted that, if the tank is partly full, the sloshing water introduces another mode of vibration which will affect the behavior of the structure.

\section{SUMMARY}

The free oscillations of a rocking block are analyzed and the rocking period and energy loss are set forth. The overturning of a block by a constant, horizontal acceleration, by a single sine pulse, and by an earthquake type excitation are examined. It is shown that there is an unexpected scale effect which makes the larger of two geometrically similar blocks more stable than the smaller block. It is also shown that the stability of a tall slender block subjected to earthquake motion is much greater than would be inferred from its stability against a constant horizontal force. In the light of these facts, the occasional survival of a slender structure that is apparently highly unstable is not surprising.

California Institute of Technology

Pasadena, California

Manuscript received April 12, 1962. 